\title{
Editorial
}

\section{Justizielle Kontrolle in der Europäischen Rechtsfabrik}

Die Europäische Union ist auf dem Weg, der Europäischen Menschenrechtskonvention beizutreten. Dies kann ein Gewinn für den Schutz der Grundrechte in Europa sein. In einem besonders grundrechtssensiblen Bereich - dem Raum der Freiheit, der Sicherheit und des Rechts - gibt es seit längerem deutliche Signale, dass die einseitige Orientierung an exekutivischen Interessen innerer Sicherheit zumindest aufgeweicht ist: Grundrechte im Strafverfahren, auch der Datenschutz sollen europaweit gestärkt werden. Die Europäische Rechtsfabrik produziert: Mehr Europäische Gesetze, die den Grundrechtsschutz zum Gegenstand haben, mehr politische Empfehlungen, den institutionellen Rechtsschutz auszubauen. Abseits der Rettungsschirme scheint die Europäische Union die „Freude des schönen Gotterfunkens“ von Freiheit und Rechtsschutz zelebrieren zu wollen. Verbreitet der Funken indes nur schönen Schein? Erfahrungen mit politischer und administrativer Macht in Europa belegen noch das Gegenteil. Die Beiträge dieses Hefts - das sich in seinem Schwerpunkt der justiziellen Kontrolle in der Europäischen Rechtsfabrik widmet - formulieren Zweifel, ob die Institutionalisierung und Implementierung justizieller Schutzmechanismen gelingt, ob sie gelingen kann und soll.

So konstatiert Henri Labayle einen Prozess der Verrechtlichung, der zwar einerseits Grundrechte im Europäischen Strafrechtsraum formell etablieren will, der aber andererseits keine hinreichende Absicherung in einem Europäischen Justizsystem hat. Die Rechtsfabrik produziert mehr Regeln, überlasst diese Regeln aber einer vielfältigen handwerklichen Pflege, die in den Mitgliedstaaten angewendet wird. Diese Art der Rechtsfabrik vergisst überwiegend - noch - den Schutz des Individuums. Erhard Denningers Beitrag thematisiert die Defizite europäischen Rechtsschutzes selbst: die Interpretation europäischer Normen ist nicht immer durch die bestmögliche Entfaltung von Individualrechten motiviert, sondern durch ,gemeinschaftsrechtspolitische Vorverständnisse“" geprägt. Systemische Integration geht darin vor individueller Freiheit. $G a-$ vin Robinson macht deutlich, dass die klassische hierarchische Form justizieller Kontrolle angesichts der Vernetzung von Daten, deren Speicherung auf Vorrat und Verarbeitung durch Dritte, scheitern muss: Soziale Kontrolle, die sich von Staaten, auch von einem Staatenverbund entkoppelt, bedarf einer Stärkung gesamteuropäischen Rechtsschutzes. Wie dringlich dieser ist, belegt der Beitrag von Sandro Dicker: die Europäische Ermittlungsanordnung will die nationalen Systeme der Beweisverbote überwinden, jedoch ohne sicherzustellen, dass in Europa überall das gleiche Niveau strafprozessualen Grundrechtsschutzes herrscht - und zwar im Detail.

Jenseits des Schwerpunkts dieses Hefts geht es ebenfalls um sehr grundrechtssensible Bereiche, die den Schutz von Individuen gegen administrative oder auch religiöse Macht zum Gegenstand haben. Dirk Wüstenberg fordert, bei der Änderung des $\S 226 \mathrm{StGB}$ das Tatbestandsmerkmal der „Genitalverstümmelung“ durch das Tatbestandsmerkmal des 
„Organs“ zu ersetzen, um den rechtlichen Vorgaben der Vereinten Nationen und der Weltgesundheitsorganisation zu entsprechen. Astrid Wallrabenstein schliesslich klagt eine verfassungskonforme Neuregelung des Asylbewerbungsleistungsgesetzes ein, die deutlich über den „Status Quo“ hinausweist.

Wir hoffen, dass dieses Heft einen kritisch-konstruktiven Beitrag zur Gesetzesproduktion in der Europäischen Rechtsfabrik zu leisten vermag.

Luxemburg, im Dezember 2012

Stefan Braum 